\title{
THE DISCRETE CORRELATION FUNCTION: A NEW METHOD FOR ANALYZING UNEVENLY SAMPLED VARIABILITY DATA
}

\author{
R. A. Edelson \\ Center for Astronomy and \\ and Space Astrophysics \\ University of Colorado \\ Boulder, CO 80309-0391 \\ U. S. A.
}

\author{
J. H. Krolik \\ Department of Physics \\ and Astronomy \\ Johns Hopkins University \\ Baltimore, MD 21218 \\ U. S. A.
}

A method of measuring correlation functions without interpolating in the temporal domain, the Discrete Correlation Function, is introduced. It provides an assumption-free representation of the correlation measured in the data, and allows meaningful error estimates. This method does not produce spurious correlations at zero lag due to correlated errors. It is shown that physical interpretation of the cross-correlation function of two series believed to be related by a convolution requires knowledge of the input function's fluctuation power spectrum. In the case of AGN line-continuum cross-correlation functions, the interpretation also involves model-dependence in the form of symmetry assumptions, and must take into account intrinsic scale bias. Application to published data for Akn 120 and NGC 4151 illustrates this method's capabilities. No correlation was found for the optical data for Akn 120, but the ultraviolet NGC 4151 data show a strong correlation, indicating that the broad C IV feature emanates from a region whose size is greater than 1.2 and less than 20 light days. These bounds on the size of the line-emitting region in NGC 4151 are in good agreement with the predictions of photoionization models.

\section{DISCUSSION}

LAWRENCE When normalising the cross correlation function, you have to use the intrinsic source variance, by removing the contribution of measurement error to the total variance. Have you made this correction?

EDELSON Yes, we have. This point was an important contribution to our understanding of the errors in correlation functions. 